\title{
Frecuencia del Polimorfismo Genético ACTN3 R577X y ECA I/D en Atletas Ciegos de Fútbol 5
}

\author{
Frequency of Genetic Polymorphism ACTN3 R577X and \\ $\mathrm{ACE}$ I/D in Blind Athletes of 5-a-Side Football
}

\author{
Glauber Lameira Oliveiraa, ${ }^{1,2,3}$; Talita Adão Perini Oliveiraa ${ }^{1,2,3}$; Ramon Pereira Souza ${ }^{2}$; Soraia Izabel \\ Corrêa Cabral'² João Rafael Valentim-Silva ${ }^{4}$; José Irineu Gorla ${ }^{5}$ \& José Fernandes Filho ${ }^{2,3}$
}

\begin{abstract}
OLIVEIRA, G. L.; OLIVEIRA, T. A. P.; SOUZA, R. P.; CABRAL, S. I. C.; VALENTIM-SILVA, J. R.; GORLA, J. I. \& FERNANDES
FILHO, J. Frecuencia del polimorfismo genético actn3 R577X y eca I/D en atletas ciegos de fútbol. Int. J. Morphol., 38(5):1336-1340, 2020.

RESUMEN: El objetivo de este estudio fue describir la frecuencia genotípica y alélica delACTN3 R577X y ECA I/D en atletas ciegos de fútbol 5. Se incluyó una metodología descriptiva con una muestra de 63 deportistas ciegos (28,0 $\pm 5,8$ años), todos varones, de equipos de fútbol 5 de alto rendimiento. El polimorfismo se determinó mediante la reacción en cadena de la polimerasa en tiempo real (RT-PCR). La estadística fue descriptiva realizada a partir de las medidas de frecuencia de genotipos y alelos. La frecuencia genotípica de laACTN3 en los deportistas presentó la siguiente distribución: el 28,6 \% con genotipo RR, el 54 \% con RX y el 17,4 \% con XX y frecuencia alélica del 55,6 \% para el alelo R y del 44,4\% para el alelo X. En cuanto a la ECA I/D, la frecuencia genotípica fue del 63,5 \% para el genotipo ID, del $22,2 \%$ para el DD y del 14,3\% para el II. La frecuencia alélica presentó prevalencia del alelo D con el 53,9\%. El estudio constató una predominancia de los genotipos y alelos representativos de las modalidades de fuerza y velocidad para ACTN3 R577X y ECA I/D de atletas de fútbol 5.
\end{abstract}

PALABRAS CLAVE: Discapacidad visual; Atletas paralímpicos; Polimorfismo.

\section{INTRODUCCIÓN}

La necesidad de optimizar el rendimiento individual de un atleta, así como el rendimiento colectivo de un equipo, ha impulsado el desarrollo de estudios orientados a la investigación de marcadores genéticos (Casilha et al., 2018). Entre los estudios de esta naturaleza, el polimorfismo 577X del gen de la alfa actina-3 (ACTN3) y el polimorfismo I/D del gen de la enzima convertidora de angiotensina (ECA) han sido investigados (Kikuchi et al., 2014; Coelho et al., 2014).

En cuanto a los polimorfismos de la ACTN3 y ECA, el predominio de los alelos $\mathrm{R}$ (genotipos RR y RX) y D (genotipos ID y DD), respectivamente, ha sido asociado con atletas que practican deportes de potencia, que requieren fuerza y velocidad. En lo que respecta a atletas de los deportes de resistencia han sido reportados el genotipo XX para ACTN3 y genotipo II para ECA, respectivamente (Jeremic et al., 2019), lo que sugiere que estos polimorfismos se asocian con un mejor rendimiento en los deportes con un requisito físico específico.

\footnotetext{
${ }^{1}$ Instituto Benjamin Constant (IBC), Rio de Janeiro, Brasil.

${ }^{2}$ Comité Paralímpico Brasileño (CPB), São Paulo, Brasil.

${ }^{3}$ Universidad Federal de Rio de Janeiro (UFRJ), Rio de Janeiro, Brasil.

${ }^{4}$ Centro Universitario Uninorte, Rio Branco, Brasil.

${ }^{5}$ Universidad Estatal de Campinas (UNICAMP), Campinas, Brasil.
}

El estudio del polimorfismo ganó notoriedad en la medicina del deporte debido a su asociación con el rendimiento deportivo y su influencia del $20 \%$ al $80 \%$ en el rendimiento de atletas (MacArthur \& North, 2007). Sin embargo, estas investigaciones han sido restringidas a los deportes olímpicos (Orysiak et al., 2014; João et al., 2015; Rocha et al., 2020) dejando una laguna de conocimiento a explorar entre modalidades paralímpicas.

Entre los deportes paralímpicos, el fútbol 5 todavía no dispone de un estudio científico sobre los marcadores genéticos incluyendo los polimorfismos de la ACTN3 y ECA y su predisposición genética para el rendimiento, lo que retrata el carácter inédito de esta investigación revistiéndola de importancia y justificando su realización. De este modo, el objetivo de este estudio fue describir la frecuencia genotípica y alélica de los polimorfismos ACTN3 R577X y ECA I/D en atletas ciegos de fútbol 5. 


\section{MATERIAL Y MÉTODO}

Muestra. Se estudiaron 63 atletas ciegos varones $(28,0 \pm$ 5,8 años) procedentes de diez diferentes equipos brasileños de fútbol 5 de alto rendimiento, federados a la Confederación Brasileña de Deportes de Discapacitados Visuales (CBDV) y clasificados funcionalmente como ciegos $\mathrm{B} 1$, los cuales poseen percepción luminosa sin la posibilidad de identificación de objeto.

El estudio contó con el consentimiento de CBDV. La participación de los atletas fue de carácter voluntario y condicionada a la firma del Término de Consentimiento Libre e Informado (TCLE), después de aclaraciones sobre los procedimientos de toma de las muestras.

Este estudio se realizó según las normas que tienen como por principio las recomendaciones de la Declaración de Helsinki para investigaciones con seres humanos y fue aprobado por el Comité de Ética e Investigación de la Universidad Federal de Río de Janeiro (CEP-HUCFF-UFRJ), obteniéndose el registro CAAE nro. 1.707.380.

Protocolos y Equipos. Para la evaluación del polimorfismo R577X del gen de la ACTN3 y ECA I/D, se utilizó material biológico procedente de muestras de sangre extraídas utilizándose lanceta y cartón FTA $®$ para la fijación de células sanguíneas y la captura del ADN en la matriz de celulosa. El material se envió al laboratorio de ADN Biotech ${ }^{\circledR}$ en Goiás-Brasil, para análisis genético.
A partir de la extracción de ADN, las muestras fueron sometidas a la técnica PCR (Reacción en Cadena de la Polimerasa) en tiempo real usando el equipo Step OneTM (Applied Biosystems, Carlsbad, California, EE.UU.).

Análisis Estadístico. Para el análisis de los datos se utilizó estadística descriptiva a partir de las medidas de frecuencia de genotipos y alelos por medio del programa Prism Stat 5.0 (Graphpad, San Diego, California, EE.UU.).

\section{RESULTADOS}

En la Tabla I se muestran las frecuencias genotípicas y alélicas de la ACTN3 en atletas ciegos de fútbol 5 de alto rendimiento que participaron en esta investigación.

En cuanto a la distribución del genotipo ACTN3, se constató que los atletas presentan mayor frecuencia para el heterocigoto RX (54\%) y alelo R $(55,6 \%)$.

En la Tabla II se muestran las frecuencias genotípicas y alélicas de la ECA I/D en atletas ciegos de fútbol 5 de alto rendimiento.

Para el análisis de la ECA, se observó predominio del genotipo ID $(63,5 \%)$ y del alelo D $(53,9 \%)$ en la muestra total y también entre atletas de todas las posiciones tácticas del juego.

Tabla I. Frecuencias genotípicas y alélicas del polimorfismo ACTN3 R577X en atletas ciegos de fútbol 5 de alto rendimiento.

\begin{tabular}{lrcccc}
\hline Posicionamiento & \multicolumn{3}{c}{ Genotipo $\mathrm{n}(\%)$} & \multicolumn{2}{c}{ Frec. Alélica n (\%) } \\
\cline { 2 - 6 } \multicolumn{1}{c}{ táctico $(\mathrm{n})$} & $\mathrm{RR}$ & $\mathrm{RX}$ & $\mathrm{XX}$ & $\mathrm{X}$ \\
\hline Cierre $(\mathrm{n}=26)$ & $8(30,8 \%)$ & $12(46,2 \%)$ & $6(23 \%)$ & $28(53,8 \%)$ & $24(46,2 \%)$ \\
Pívot $(\mathrm{n}=13)$ & $2(15,4 \%)$ & $8(61,5 \%)$ & $3(23,1 \%)$ & $12(46,2 \%)$ & $14(53,8 \%)$ \\
Ala $(\mathrm{n}=24)$ & $8(33,3 \%)$ & $14(58,3 \%)$ & $2(8,3 \%)$ & $30(62,5 \%)$ & $18(37,5 \%)$ \\
General $(\mathrm{n}=63)$ & $18(28,6 \%)$ & $34(54 \%)$ & $11(17,4 \%)$ & $70(55,6 \%)$ & $56(44,4 \%)$ \\
\hline
\end{tabular}

Tabla II. Frecuencias genotípicas y alélicas del polimorfismo ECA I/D en atletas ciegos de fútbol 5 de alto rendimiento.

\begin{tabular}{lcccccc}
\hline \multicolumn{2}{c}{\begin{tabular}{c} 
Posicionamiento \\
\multicolumn{2}{c}{ táctico (n) }
\end{tabular}} & DD & Genotipo n (\%) & \multicolumn{2}{c}{ Frec. Alélica n (\%) } \\
\hline Cierre & $(\mathrm{n}=26)$ & $8(30,8 \%)$ & $11(42,3 \%)$ & $7(26,9 \%)$ & $27(51,9 \%)$ & $25(48,1 \%)$ \\
Pívot & $(\mathrm{n}=13)$ & $3(23,1 \%)$ & $8(61,5 \%)$ & $2(15,4 \%)$ & $14(53,8 \%)$ & $12(46,2 \%)$ \\
Ala & $(\mathrm{n}=24)$ & $9(37,5 \%)$ & $11(45,8 \%)$ & $4(16,7 \%)$ & $29(60,4 \%)$ & $19(39,6 \%)$ \\
General $(\mathrm{n}=63)$ & $14(22,2 \%)$ & $40(63,5 \%)$ & $9(14,3 \%)$ & $68(53,9 \%)$ & $58(46,1 \%)$ \\
\hline
\end{tabular}

\section{DISCUSIÓN}

Estudios que caracterizan el genotipo de la ACTN3 como factor de rendimiento físico señalan que, la predominancia del alelo R, con expresión del gen ACTN3 RR y RX como obtenido con mayor prevalencia para los 
atletas participantes de esta investigación, indican mayor rendimiento en modalidades que requieren velocidad y fuerza, en comparación con los atletas que presentan el genotipo XX (Vincent et al., 2010; Heffernan et al., 2016). Investigaciones han demostrado que el genotipo XX, a su vez, ha favorecido el rendimiento de los atletas en aquellas actividades caracterizadas como de resistencia (Eynon et al., 2009).

Souza et al. (2016) al analizar la intensidad de esfuerzo de los atletas de fútbol 5 de la selección brasileña que participaron en los Juegos Paralímpicos de Londres 2012 $(\mathrm{n}=8 ; 23,8 \pm 3,3$ años), demostraron que estos atletas permanecieron $52,4 \%$ del tiempo total de un partido (50 min) con una intensidad de esfuerzo entre las zonas 2 y 3 , de moderada a intensa, alcanzando una frecuencia cardíaca media del 89,8 \% de la frecuencia cardíaca máxima. Los autores concluyeron que la intensidad de esfuerzo en esta modalidad es intermitente y de corta duración, donde la obtención de la velocidad máxima en corto período de tiempo favorece el rendimiento.

Un hallazgo importante de este estudio que corrobora la investigación de Souza et al., está relacionado con el aumento de la frecuencia del ACTN3-RX y disminución de la frecuencia del ACTN3-XX, reflejando la característica de esta modalidad, de potencia anaeróbica, en la que la velocidad y la fuerza son determinantes para el buen rendimiento.

Cuando se evalúan los resultados obtenidos por los atletas en las diferentes posiciones tácticas, se nota que el genotipo descrito también sugiere la predisposición de estos atletas a un buen rendimiento en esta modalidad, debido a la predominancia del genotipo heterocigoto ACTN3-RX en todas ellas (pivotes $=61,5 \%$, alas $=58,3 \%$ y cierres $=$ $46,2 \%)$

Altas frecuencias del genotipo ACTN3-RR/ACTN3RX también se han encontrado en la literatura para modalidades en que predominan la fuerza y potencia (MacArthur \& North; Gómez-Gallego et al., 2009) y del ACTN3-XX en deportes donde la resistencia es determinante para el rendimiento (Eynon et al.). Un estudio desarrollado por Vincent $e t$ al. (2007), ha demostrado que la expresión de la ACTN3 está relacionada con los músculos de contracción rápida para producción de fuerza y alta velocidad en individuos con alelo R.

Un factor importante en los hallazgos del presente estudio está relacionado no sólo con la frecuencia del alelo $\mathrm{R}$, que confiere fuerza y potencia, sino también con el hecho del genotipo prevalente entre atletas de todas las posiciones, ser heterocigoto (RX) de dominio parcial, el cual, según Coelho et al. (2014) representa un carácter mixto, indicando que la resistencia (alelo X) también puede contribuir al rendimiento. Como los partidos de fútbol 5 tienen una duración total de 50 minutos, este factor también puede permitir un mejor rendimiento entre estos atletas durante los partidos.

Resultados similares fueron obtenidos por Pimenta et al. (2013), donde el $89 \%$ de la muestra $(\mathrm{n}=178)$ de atletas de fútbol de campo presentaron el alelo R (genotipo RX o $\mathrm{RR}$ ). Similarmente al fútbol 5, en las acciones motoras específicas del fútbol de campo, la realización de carreras cortas asociados a gran velocidad de reacción puede determinar el buen desempeño en el juego.

Otro estudio realizado también con fútbol de campo (Coelho et al., 2014), pero comparando los genotipos entre diferentes niveles competitivos, encontró que los atletas presentaron mayor frecuencia para el alelo dominante R, independientemente del nivel amateur, infantil y profesional.

Estudios realizados con otras modalidades, con características similares al fútbol 5 , de intensidad intermitente, presentaron también distribución genotípica con prevalencia del ACTN3-RX o ACTN3-RR entre atletas brasileños (Ribas et al., 2017), japoneses (Kikuchi et al.), rusos (Fiuza-Luces et al., 2011), israelíes (Eynon et al.), españoles (Rodríguez-Romo et al., 2013), africanos (Yang et al., 2007), australianos (Yang et al., 2003) y polacos (Orysiak et al.) apuntando, de hecho, hacia la misma dirección.

En cuanto a la deficiencia de alfa actinina-3 (ACTN3$\mathrm{XX}$ ), que se asocia directamente con una disminución en el rendimiento de las fibras musculares de contracción rápida (Castilha et al.), se observó una baja frecuencia entre los atletas participantes de esta investigación $(17,4 \%)$, lo que también indica predisposición de estos atletas a satisfacer las exigencias de intensidad intermitente (Souza et al.), propia de esta modalidad.

Considerando la distribución de frecuencia genotípica de ACTN3-RR + ACTN3-RX, la cual se refiere al dominio del alelo $\mathrm{R}$, los atletas pertenecientes a la posición ala presentaron mayor frecuencia ( $91,6 \%$ ), seguidos de los cierres (77 \%) y pivotes $(76,9 \%)$, lo que puede ser justificado por el requisito pertinente a cada posicionamiento táctico en el juego, en el que los alas tienen mayor densidad e intensidad de acciones que cierres y pivotes (Gorla et al., 2017).

Independientemente de cada una de las posiciones tácticas en el juego, este estudio constató que el perfil genotípico ACTN3-RX, seguido de ACTN3-RR, es más frecuente entre los atletas ciegos de fútbol 5 , lo que indica que 
estos atletas tienen predisposición genética para rendimiento en las actividades con prevalencia del metabolismo anaeróbico, las cuales demandan estímulos intensos y corta duración en máxima velocidad (Orysiak et al.).

El polimorfismo de la ECA, también señalado como factor genético que influye en el rendimiento deportivo (Ribas et al.), presentó mayor frecuencia para el genotipo ID $(63,5 \%)$, seguido del genotipo DD $(22,2 \%)$ y II $(14,3$ $\%)$, tanto para la muestra general de atletas, como en los diferentes posicionamientos tácticos en el juego (Tabla II). Estos datos corroboran los de otros estudios que también han reportado el genotipo ID más prevalente para atletas de rugby $(49,7 \%$ ) (Heffernan et al.), futsal (43\%), (Rocha et al.) y fútbol $(54,8 \%)$ (Rocha et al.); (57,5\%) (Salgueirosa et al., 2017), evidenciando su presencia entre deportes en los que la fuerza y velocidad juegan un papel más grande en la determinación del éxito (Jeremic et al.).

Cuando sumadas las frecuencias genotípicas de ACE $\mathrm{DD}$ e ID, a partir de la presencia del alelo D, se verificó que el $85,7 \%$ de los atletas presentaron tal predisposición genética, comportamiento similar considerando los posicionamientos tácticos en el juego (pivote $=84,6 \%$; ala $=$ $83,3 \%$; cierre $=73,1 \%$ ).

La prevalencia de la frecuencia alélica para el alelo D $(53,9 \%)$ obtenida entre los atletas de esta investigación, considerando tanto la muestra general como en los diferentes posicionamientos tácticos en el juego (ala=60,4\%; pívot $=53,8 \%$; cierre $=51,9 \%$ ) corrobora la literatura (Hefferman et al., 2016; Papadimitriou et al., 2016), que asocia su expresión a los deportes de fuerza y velocidad, como en el fútbol 5 (Gorla et al.). Estos hallazgos pueden representar una ventaja entre estos atletas, ya que disponen de una predisposición genética capaz de atender a las especialidades de esta modalidad.

A partir de la evaluación genética de atletas de fútbol 5, esta investigación llena la brecha existente en la literatura, siendo la pionera en describir la frecuencia genotípica y alélica de la ACTN3 y de la ECA I/D en atletas ciegos de fútbol 5 , con el fin de ofrecer subsidios a la orientación deportiva y a la planificación de programas de entrenamiento más específicos y eficientes para atletas de esta modalidad, principalmente cuando existen diferencias posicionales tácticas intradeportivas.

Sin embargo, la descripción de la frecuencia genotípica y alélica para estos marcadores puede contribuir con una parte del éxito de la planificación del entrenamiento del atleta, pues que otros factores biológicos y ambientales también deben ser considerados.
La principal limitación de este estudio es la escasez de estudios de esta naturaleza en esta modalidad, sin embargo, este hecho, demuestra el carácter inédito de este trabajo.

Los resultados de este estudio, con predominio del genotipo RX y del alelo R para ACTN3 y del genotipo ID y del alelo D para ECA, indican que los atletas de fútbol 5 poseen una predisposición genética característica de atletas de deportes de potencia, con exigencia de fuerza y velocidad para el desarrollo de las actividades, lo que combina con las necesidades específicas de esta modalidad.

Finalmente, se sugiere que nuevas investigaciones con ACTN3 y ECA y sus polimorfismos se realicen con otras modalidades deportivas paralímpicas para conocer la predisposición genética de sus atletas, subsidiando el entrenamiento para el alto rendimiento deportivo.

\section{AGRADECIMIENTOS}

Comité Paralímpico Brasileño (CPB) y Confederación Brasileña de Deportes para Discapacitados Visuales (CBDV).

OLIVEIRA, G. L.; OLIVEIRA, T. A. P.; SOUZA, R. P.; CABRAL, S. I. C.; VALENTIM-SILVA, J. R.; GORLA, J. I. \& FERNANDES FILHO, J. Frequency of Genetic Polymorphism ACTN3 R577X and ACE I/D in Blind Athletes of 5-a-side football. Int. J. Morphol., 38(5):1336-1340, 2020.

SUMMARY: The aim of this study was to describe the genotypic and allele frequency of ACTN3 R577X and ACE I/D in blind athletes of 5-a-side football performance. A descriptive methodology was included with a sample of 63 blind male athletes $(28.0 \pm 5.8$ years $)$ of football teams with a 5 -a-side performance rating. The polymorphism was determined by means by of real-time Polymerase Chain Reaction (rt-PCR). Statistics were descriptive based on the measures of frequency of genotypes and alleles. The genotypic frequency of ACTN3 by the athletes presented the following distribution: $28.6 \%$ with RR genotype, $54 \%$ with RX and $17.4 \% \mathrm{XX}$ and allele frequency of $55.6 \%$ for the R allele and $44.4 \%$ for the X allele. As for ACE I/D, the genotype frequency was $63.5 \%$ for genotype ID, $22.2 \%$ for DD and $14.3 \%$ for II. The allele frequency showed a predominance of the $\mathrm{D}$ allele with $53.9 \%$. The study found for ACTN3 R577X and ACE I/ $\mathrm{D}$ of blind athletes of 5-a-side football, a predominance of genotypes and alleles representative of strength and speed modalities.

KEY WORDS: Visual impairment; Paralympic; Polymorphism. 


\section{REFERENCIAS BIBLIOGRÁFICAS}

Coelho, D. B.; Pimenta, E. M.; Rosse, I. C.; Carvalho, M. R. S.; Costa, G. G. \& Silami-Garcia, E. Comparison of the genotypes and allele frequencies of ACTN3 of football players from different categories. Rev. Bras. Cienc. Mov., 22(4):97-106, 2014.

Castilha, F. A.; Ferreira, H. R.; Oliveira, G. L.; Oliveira, T. A. P.; Fernandes, P. R. \& Fernandes Filho, J. The influence of gene polymorphysms and genetic markers in the modulation of sports performance: a review. $J$. Exerc. Physiol. Online, 21(2):248-64, 2018.

Eynon, N.; Duarte, J. A.; Oliveira, J.; Sagiv, M.; Yamin, C.; Meckel, Y.; Sagiv, M. \& Goldhammer, E. ACTN3 R577X polymorphism and israeli top-level athletes. Int. J. Sports Med., 30(9):695-8, 2009.

Fiuza-Luces, C.; Ruiz, J. R.; Rodríguez-Romo, G.; Santiago, C.; GómezGallego, F.; Yvert, T.; Cano-Nieto, A.; Garatachea, N.; Morán, M. \& Lucia, A. Are 'endurance' alleles 'survival' alleles? Insights from the ACTN3 R577X polymorphism. PloS One, 6(3):e17558, 2011.

Gómez-Gallego, F.; Santiago, C.; González-Freire, M.; Muniesa, C. A.; Fernández Del Valle, M.; Pérez, M.; Foster, C. \& Lucia, A. Endurance performance: genes or gene combinations? Int. J. Sports Med., 30(1):66$72,2009$.

Gorla, J. I.; Silva, A. A. C.; de Campos, L. F. C. C.; dos Santos, C. F.; de Almeida, J. J. G.; Duarte, E. \& Queiroga, M. R. Composição corporal e perfil somatotípico de atletas da seleção brasileira de futebol de 5 . Rev. Bras. Cienc. Esporte, 39(1):79-84, 2017.

Heffernan, S. M; Kilduff, L. P.; Erskine, R. M.; Day, S. H.; McPhee, J. S.; McMahon, G. E.; Stebbings, G. K.; Neale, J. P. H.; Lockey, S. J.; Ribbans, W. J.; et al. Association of ACTN3 R577X but not ACE I/D gene variants with elite rugby union player status and playing position. Physiol. Genomics, 48(3):196-201, 2016.

Jeremic, D.; Macuzic, I. Z.; Vulovic, M.; Stevanovic, J.; Radovanovic, D.; Varjacic, V. \& Djordjevic, D. ACE/ACTN3 genetic polymorphisms and athletic performance of female soccer players. Rev. Bras. Med. Esporte, 25(1):35-9, 2019.

João, A. F.; Vargas, A. C.; Mosqueira, C. H.; da Silva, S. F.; Izquierdo, M.; Mella, H. S.; Ramirez-Campillo, R. \& Fernandes Filho, J. Gene polymorphism ACTN3 and ACE in selected gymnasts athletes in Brazil and Japan. Int. J. Morphol., 33(1):262-6, 2015.

Kikuchi, N.; Nakazato, K.; Min, S. K.; Ueda, D. \& Igawa, S. The ACTN3 R577X polymorphism is associated with muscle power in male japanese athletes. J. Strength Cond. Res., 28(7):1783-9, 2014.

MacArthur, D. G. \& North, K. N. ACTN3: a genetic influence on muscle function and athletic performance. Exerc. Sport Sci. Rev., 35(1):30-4, 2007.

Orysiak, J.; Busko, K.; Michalski, R.; Mazur-Róz ycka, J.; Gajewski, J.; Malczewska-Lenczowska, J.; Sitkowski, D. \& Pokrywka, A. relationship Between ACTN3 R577X polymorphism and maximal power output in elite Polish athletes. Medicina (Kaunas), 50(5):303-8, 2014.

Papadimitriou, I. D.; Lucia, A.; Pitsiladis, Y. P.; Pushkarev, V. P.; Dyatlov, D. A.; Orekhov, E. F.; Artioli, G. G.; Guilherme, J. P. L. F.; Lancha Jr., A. H.; Gineviciene, V.; et al. ACTN3 R577X and ACE I/D gene variants influence performance in elite sprinters: a multi-cohort study. $B M C$ Genom., 17:285, 2016.

Pimenta, E. M.; Coelho, D. B.; Veneroso, C. E.; Coelho, E. J. B.; Cruz, I. R.; Morandi, R. F.; Pussieldi, G. D. A.; Carvalho, M. R. S.; Garcia, E. S. \& De Paz Fernández, J. A. Effect of ACTN3 gene on strength and endurance in soccer players. J. Strength Cond. Res., 27(12):3286-92, 2013.

Pontes, I. M. \& Astolfi Filho, S. Frequency of gene ACE I polymorphism I-D in athletes of different sports. Rev. Bras. Med. Esporte, 26(2):107$12,2020$.

Ribas, M. R.; Oliveira Netto, Z. C.; Salgueirosa, F.; Fernandes, F.; de Matos, O. \& Bassan, J. C. Associação dos polimorfismos actn3 r577x e ace i/ d em lutadores Brasileiros. Rev. Bras. Med. Esporte, 23(6):469-72, 2017.
Rocha, A. W. O.; do Nascimento, W. M.; Oliveira, C. M. C.; Neto, J. M. P.; Nascimento, O. V.; dos Santos, J. O. L.; Viera, E. P.; Brunetta, H. S.; Rodríguez-Romo, G.; Yvert, T.; de Diego, A.; Santiago, C.; Díaz De Durana, A. L.; Carratalá, V.; Garatachea, N. \& Lucia, A. No association between ACTN3 R577X polymorphism and elite judo athletic status. Int. J. Sports Physiol. Perform., 8(5):579-81, 2013.

Salgueirosa, F. M.; Rodrigues, P.; Seniski, G. G.; Wharton, L. \& Osiecki, R. ACTN3 R577X and ACE I/D genotype frequencies of professional soccer players in Brazil. J. Exerc. Physiol. Online, 20(6):129-38, 2017.

Souza, R. P.; Alves, J. M. V. M.; Gorla, J. I.; Novaes, G.; Cabral, S. I. C.; Neves, E. B. \& Nogueira, C. D. Characterization of the intensity of effort of blind athletes from the Brazilian Football 5-A-Side national team. J. Health Biol. Sci., 4(4):218-26, 2016.

Vincent, B.; De Bock, K.; Ramaekers, M.; Van den Eede, E.; Van Leemputte, M.; Hespel, P. \& Thomis, M. A. ACTN3 (R577X) genotype is associated with fiber type distribution. Physiol. Genomics, 32(1):58-63, 2007.

Vincent, B.; Windelinckx, A.; Nielens, H.; Ramaekers, M.; Van Leemputte, M.; HespeL, P. \& Thomis, M. A. Protective role of alpha-actinin-3 in the response to an acute eccentric exercise bout. J. Appl. Physiol., 109(2):564-73, 2010

Yang, N.; MacArthur, D. G.; Gulbin, J. P.; Hahn, A. G.; Beggs, A. H.; Easteal, S. \& North, K. ACTN3 genotype is associated with human elite athletic performance. Am. J. Hum. Genet., 73(3):627-31, 2003.

Yang, N.; MacArthur, D. G.; Wolde, B.; Onywera, V. O.; Boit, M. K.; Lau, S. Y. M. A.; Wilson, R. H.; Scott, R. A.; Pitsiladis, Y. P. \& North, K. The ACTN3 R577X polymorphism in East and West African athletes. Med. Sci. Sports Exerc., 39(11):1985-8, 2007.

Dirección para correspondencia

Glauber Lameira Oliveira

Instituto Benjamin Constant

Avenida Pasteur, 350/368

CEP: 22290240

Rio de Janeiro - RJ

BRASIL

E-mail: lameiraglauber@gmail.com

Recibido : $15-04-2020$

Aceptado: 01-06-2020 